\section{Fluid therapy under anaesthesia: are we giving too much?}

\section{Ian Self}

Intravenous fluid therapy is recommended for any anaesthetised patient undergoing an interventional procedure. The aim of intravenous fluids is to maintain circulating volume to ensure adequate oxygen delivery and perfusion to organs. Reasons put forward for the administration of fluids under anaesthesia include the potential fluid deficits caused by perioperative fasting, the vasodilatory effects of anaesthetic drugs leading to a relative hypovolaemia, and the losses from the respiratory tract (worsened by endotracheal intubation) and the surgical site (evaporative, third space or haemorrhage). Traditionally, it has been recommended that intravenous crystalloids are administered at doses between 5 and $20 \mathrm{ml} / \mathrm{kg} / \mathrm{hr}$, although there is limited evidence that these rates achieve improved clinical outcomes.

Recently, the traditional dose approach to fluid therapy has been questioned, and there are several studies which show no benefits of higher volume fluid administration compared to more restricted volumes. Indeed, the CEPSAF study (Brodbelt et al., 2007) showed that cats receiving fluids were more likely to die under anaesthesia and it was thought that fluid overload in these patients could be a contributing factor.

In order to administer effective fluid therapy, current opinion is turning towards individualised fluid therapy for each patient and adoption of a goal-directed approach. For this approach to be successful, effective monitoring of intravascular volume status is necessary using a combination of clinical parameters including mucous membrane colour, capillary refill time, pulse quality and rate, urine output, respiratory wheezes or crackles and moistness of mucous membranes. Additionally, monitors such as blood pressure and pulse oximeters can be used to obtain objective data. When taken in combination, the clinical signs can be used to assess the adequacy of fluid administration and whether the patient's fluid balance is being adversely affected by over- or under-administration. Similarly, in goal-directed therapy the administration of fluids can be altered to maintain the desired clinical parameter within normal limits; for example to maintain urine output at or above $1 \mathrm{ml} / \mathrm{kg} / \mathrm{hr}$ in patients with renal disease.

The most recent developments in fluid therapy monitoring have examined the dynamic responsiveness of parameters such as pulse pressure variation and plethysmographic waveform (pulse oximeter) variations in response to fluid therapy. This is achieved in patients being mechanically ventilated who have no arrhythmias, where alterations in the depression of the waveforms during inspiration before and after fluid therapy can be predictors of intravascular volume status.

\section{FURTHER READING}

Brodbelt, DC; Pfeiffer, DU; et al. Risk factors for anaestheticrelated death in cats: results from the confidential enquiry into perioperative small animal fatalities (CEPSAF). British Journal of Anaesthesia 2007; 99: 617-623.

\section{KEY LEARNING OBJECTIVES}

- Understand the clinical reasons for fluid administration in anaesthetised patients

- Describe the concept of 'goal-directed therapy' in fluid administration

- Recognise the principles of dynamic fluid-

responsiveness monitoring techniques

\section{MULTIPLE CHOICE QUESTIONS}

1. Which is the most commonly used crystalloid for fluid therapy in anaesthetised patients?
a. Hartmann's solution
b. Oxyglobin solution
c. Hydroxyethyl starch
d. Fresh frozen plasma

2. How may renal perfusion be directly assessed in anaesthetised patients?
a. Mucous membrane colour
b. Capillary refill time
c. Arterial blood pressure
d. Urine output

3. Acute over-infusion of fluids in cats commonly manifests as which clinical sign?
a. Central nervous system swelling leading to seizure activity
b. Reduced hepatic function
c. Pulmonary oedema leading to crackles
d. Development of urinary cystic calculi

4. Pulse pressure variation is a dynamic fluidresponsiveness test used in anaesthetised patients. Under what specific conditions can it be assessed?
a. Spontaneous respiration, no cardiac arrhythmias
b. Spontaneous respiration, occasional cardiac arrhythmias
c. Controlled respiration, no cardiac arrhythmias
d. Controlled respiration, occasional cardiac arrhythmias

5. Fluid therapy in anaesthetised patients has been advocated as many anaesthetic drugs cause which of the following?
a. Hypertension
b. Vasodilation
c. Tachycardia
d. Tachypnoea 